\title{
RNA interference targeting virion core protein ORF095 inhibits Goatpox virus replication in Vero cells
}

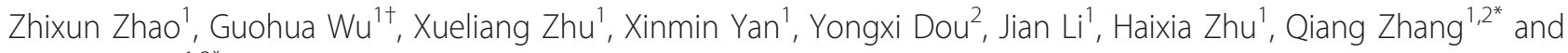
Xuepeng $\mathrm{Cai}^{1,2^{*}}$

\begin{abstract}
Background: Goatpox is an economically important disease in goat and sheep-producing areas of the world. Many vaccine strategies developed to control the disease are not yet completely successful. Hairpin expression vectors have been used to induce gene silencing in a large number of studies on viruses. However, none of these studies has been attempted to study GTPV. In the interest of exploiting improved methods to control goat pox, it is participated that RNAi may provide effective protection against GTPV. In this study we show the suppression of Goatpox virus (GTPV) replication via knockdown of virion core protein using RNA interference.

Results: Four short interfering RNA (siRNA) sequences (siRNA-61, siRNA-70, siRNA-165 and siRNA-296) against a region of GTPV ORF095 were selected. Sense and antisense siRNA-encoding sequences separated by a hairpin loop sequence were designed as short hairpin RNA (shRNA) expression cassettes under the control of a human U6 promoter. ORF095 amplicon was generated using PCR, and then cloned into pEGFP-N1 vector, named as p095/ EGFP. p095/EGFP and each of the siRNA expression cassettes (p61, p70, p165 and p296) were co-transfected into BHK-21 cells. Fluorescence detection, flow cytometric analysis, retro transcription PCR (RT-PCR) and real time PCR were used to check the efficiency of RNAi. The results showed that the ORF095-specific siRNA-70 effectively downregulated the expression of ORF095. When Vero cells were transfected with shRNA expression vectors (p61/GFP, p70/GFP, p165/GFP and p296/GFP) and then infected with GTPV, GTPV-ORF095-70 was found to be the most effective inhibition site in decreasing cytopathic effect (CPE) induced by GTPV. The results presented here indicated that DNA-based siRNA could effectively inhibit the replication of GTPV (approximately 463. 5-fold reduction of viral titers) on Vero cells.
\end{abstract}

Conclusions: This study demonstrates that vector-based shRNA methodology can effectively inhibit GTPV replication on Vero cells. Simultaneously, this work represents a strategy for controlling goatpox, potentially facilitating new experimental approaches in the analysis of both viral and cellular gene functions during of GTPV infection.

Keywords: RNAi, shRNA, ORF095, Goatpox virus

\footnotetext{
* Correspondence: qzhang1616@sohu.com; caixp@VIP.163.com

† Contributed equally

${ }^{1}$ Key Laboratory of Animal virology of the Ministry of Agriculture, State Key Laboratory of Veterinary Etiological Biology, Lanzhou Veterinary Research Institute, Chinese Academy of Agriculture Sciences, No. 1 Xujiaping, Lanzhou, Gansu 730046, PR China

Full list of author information is available at the end of the article
} 


\section{Background}

GTPV is a member of the Genus Capripoxvirus of the family Poxviridae [1], which also includes the Sheeppox virus (SPPV) and the Lumpy Skin Disease Virus (LSDV) of cattle. Both sheeppox and goatpox are endemic in Africa, the Middle East and many countries in Asia, and the diseases caused by these viruses have a significant economic impact on the livestock industry in Africa and Asia [2]. GTPV genome is approximately $150 \mathrm{kbp}$ double-stranded DNA, which composes at least 147 putative genes, including conserved replicative and structural genes and genes likely involved in virulence and host range [3]. ORF095 encodes the virion protein which constitutes a great part of the total protein content of the virion and is essential during the assembly and disassembly of virion. It is similar to myxoma virus (MYXV) M093L (accession no.AF170726) [4,5] and vaccinia virus (VACV) A4L (accession no.M35027) that encodes a $39 \mathrm{kDa}$ acidic protein, a part of the viral core, and is synthesized at late stages after infection [6,7].

RNA-mediated interference (RNAi) is a conserved gene-silencing mechanism, where by the doublestranded RNA matching is used as a signal to trigger the sequence-specific degradation of homologous mRNA [8]. RNAi can be triggered by chemically synthesised and enzymatically produced 21-25 nt long RNA duplexes in mammalian cells $[9,10]$. Since the effect of short interfering RNAs (siRNAs) is generally temporal in transfected animal cells, small RNA expression vectors have been developed to induce long-lasting RNA silencing in mammalian cells [11-14]. RNAi represents a new antiviral method and is being increasingly used to inhibit the replication of viral pathogens [15] such as foot-and-mouth disease $[16,17]$, porcine reproductive and respiratory syndrome virus [18], Newcastle disease virus [19], classical swine fever virus [20] and Monkeypox virus [21]. Hairpin expression vectors have been used to induce gene silencing in a large number of studies on viruses [11,22-26].

This study provides not only an experimental basis for the development of a new anti-GTPV strategy, but also for a new approach to the study of GTPV infection and replication.

\section{Materials and methods Viruses and cells}

Goatpox virus strain A/Goat/Qinghai/AV40/2006(a celladapted strain) was used in this study and maintained in African green monkey kidney cells (Vero). Baby Hamster Kidney cells (BHK-21) and the GTPV permissive cell line Vero (Lanzhou Veterinary Research Institute, Chin) were cultured in Dulbecco's modified Eagle's medium (DMEM; Sigma) supplemented with 10\% heat- inactivated fetal bovine serum (FBS; Hangzhou, China), $100 \mathrm{U} / \mathrm{ml}$ penicillin and $100 \mu \mathrm{g} / \mathrm{ml}$ streptomycin (Sigma). Cultures were incubated at $37^{\circ} \mathrm{C}$ with $5 \% \mathrm{CO} 2$.

\section{Construction of plasmids}

The cDNA cassettes corresponding to the conserved gene of the GTPV genome was cloned into the pEGFPN1 vector (Figure 1A). Directed cloning PCR was used to amplify the ORF095 gene, using the following primers (sense: 5'-GTCCTCGAGATGGACTTCATGAAAAAATATACTAA-3' and antisense: 5'GCGGATCCTTGCTGTTATTATCATCTAGTTTG-3') used for amplification contained the target sequences for XhoI (CTCGAG) and BamHI (GGATCC) incorporated at the 5 ' of the viral complementary sequence. Forward primer contained an ATG sequence, before the sequence that codified for the protein, as a start initiation codon of protein translation. The reverse primer uncontained a TTA sequence, which was used in the characterization of ORF095 protein and EGFP coexpression. PCR products were digested with $\mathrm{XhoI}$ and BamHI, and cloned into the pEGFP-N1 expression vector (Invitrogen, Inc., Shanghai, China). The final construct p095/EGFP (Figure 1A) was analyzed by restriction digestion and sequenced. Plasmids used for

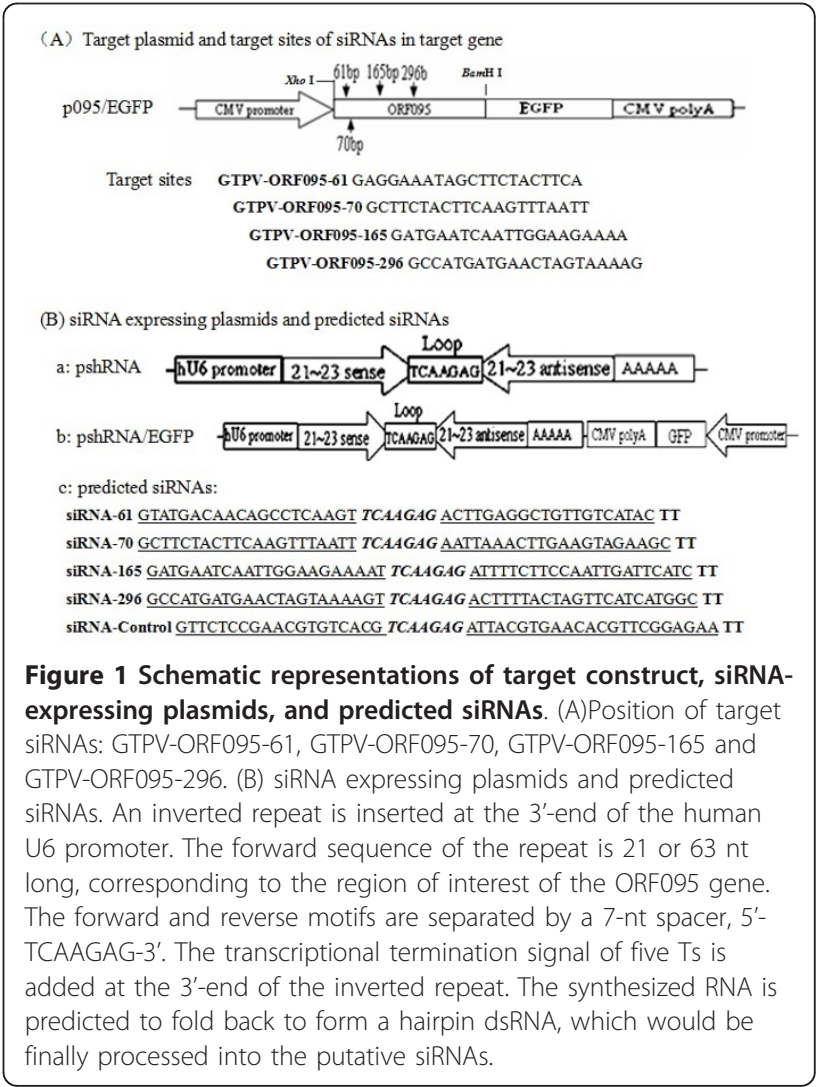


transfection were purified with the Wizard Purefection TM Plasmid DNA Purification System (Promega, USA) and quantified by Biophotometer (Eppendorf, Germany).

\section{Target sequence selection and vector construction}

As the AAGN18 $8_{U U}$ sequence ( $\mathrm{N}$, any nucleotide) has been found to be preferred for siRNA-mediated gene silencing under the control of the PolU6 promoter [27], we searched for this sequence in the ORF of ORF095 gene. Four fragments (ORF095-61, ORF095-70, ORF095-165 and ORF095-296) in the coding region of ORF095 gene were selected according to the web-based criteria (http://www.ambion.com). These selected sequences were then submitted to a BLAST searching against human genome sequence to check whether or not these potential targets have homologues in the human genome was not targeted. To construct hairpin siRNA expression cassette, the following DNA oligonucleotides were synthesized: GTPV-ORF095-61, GTPVORF095-70, GTPV-ORF095-165 and GTPV-ORF095296 (Figure 1A). The $21 \mathrm{nt}$ target sequences served as a basis for the design of the two complementary 5153 mer siRNA template oligonucleotides that were synthesized, annealed, and inserted into BamHI and $B b s I$ sites of the siRNA expression vectors pGPU6/Neo and pGPU6/GFP/Neo (GenePharma Co., Ltd, Shanghai, China), respectively. The recombinant plasmids were designated as p61, p61/GFP, p70, p70/GFP, p165, p165/ GFP, p296 and p296/GFP. The pC and pC/GFP negative controls (GenePharma Co., Ltd, Shanghai, China) were negative control plasmid, which encode hairpin siRNA that does not have homologues in mice, human and Carpripoxvirus genome databases.

\section{Transfection of the siRNA expression cassettes into BHK- 21 cells}

BHK-21 cells were seeded in six-well plates and cultured at $37^{\circ} \mathrm{C}$ and $5 \% \mathrm{CO} 2$ overnight. When the cells showed $70-80 \%$ confluence, $2.5 \mu \mathrm{g}$ of p61, p70, p165 or p296 each were cotransfected with an equal amount of p095/ EGFP using FuGENE HD Transfection Reagent (Roche, Germany) according to the Manufacturer's recommendations, respectively. Simultaneously, $2.5 \mu \mathrm{g}$ of p095/ EGFP were cotransfected with $2.5 \mu \mathrm{g}$ of $\mathrm{pC}$. Non-transfected BHK-21 cells were also used as a control.

\section{Analysis of EGFP expression in BHK-21 cells and flow cytometry assay}

After an additional $24 \mathrm{~h}$ of incubation, cells were observed for the expression of green fluorescent protein in the transfected cells was monitoring fluorescent microscope (Olympus, Japan).

Cells were further subjected to fluorescence-activated cell sorting (FACS). At $48 \mathrm{~h}$ posttransfection, the transfected cells and the controls were washed gently in phosphate-buffered saline (PBS), trypsinized and resuspended in PBS. EGFP positive cells and EGFP expression signal were evaluated by the FACS Calibur Flow Cytometry System (Becton Dickinson, USA).

\section{Reverse transcription (RT)-PCR}

To confirm the efficacy of RNAi, RT-PCR was used to amplify the target gene in the transfected cells. Total RNA was extracted from BHK-21 culture with Trizol reagent (TaKaRa, Dalian), and incubated for $1 \mathrm{~h}$ at $37^{\circ} \mathrm{C}$ with Dnase RQ1 (TaKaRa, Dalian). To detect ORF095 mRNA expression in BHK-21 cells, RT-PCR was conducted using $1.8 \mu \mathrm{g}$ of RNA extracts with Superscript one-step RT-PCR system (Gibco, BRL). Retrotranscription $\beta$-actin as a control was also amplified using the Primers 5'-CACCCGCGAGTACAACCTTC-3' (sense) and 5'-CCCATACCCACCATCACACC-3' (antisense). PCR was run for 30 cycles with $95^{\circ} \mathrm{C}$ for $30 \mathrm{~s}, 56^{\circ} \mathrm{C}$ for $45 \mathrm{~s}$ and $72^{\circ} \mathrm{C}$ for $45 \mathrm{~s}$. To verify primer specificity, a melting curve was analyzed, and RT-PCR products were further cloned into pMD18-T for sequencing.

\section{Real-time PCR analysis}

In order to use full-length ORF095 gene as a quantitative RT-PCR standard, Selected primer sequences were CTGTCTACATGATTAACCCACTCGTTCTTC (ORF095 FP primer), and GAAGTCGACTAC CCCTCTCCCTATCAGGGTCATC (ORF095 RP primer). One additional primer was synthesized for quantification of the ORF095 in real-time PCR: 5-FAMCCTTGCTCGCGAATTTCTCACCGATAMRA- 3 (TaqMan probe). The target region of real time RT-PCR was 263-389 bp of ORF095.

For quantitative analysis of the ORF095 gene, $100 \mathrm{ng}$ total RNA from p095/EGFP-transfected cells was mixed with $1 \mu \mathrm{L}$ ORF095 primer, heated to $65^{\circ} \mathrm{C}$ for $5 \mathrm{~min}$ and chilled on ice for $2 \mathrm{~min}$. To this primer template mix was added $5 \times$ buffer $(4 \mu \mathrm{L}), 10 \mathrm{mmol} / \mu \mathrm{L}$ dNTP $(1$ $\mu \mathrm{L})$, RNasin $(1 \mu \mathrm{L})$, AMV reverse transcriptase $(1 \mu \mathrm{L}$, Promega, USA) and $\mathrm{ddH}_{2} \mathrm{O}$ to a total volume of $20 \mu \mathrm{L}$. The reaction mixture was incubated at $42^{\circ} \mathrm{C}$ for $45 \mathrm{~min}$, followed by inactivation of reverse transcriptase at $75^{\circ} \mathrm{C}$ for $15 \mathrm{~min}$. Real-time PCR was performed with the ABI PRISM 7000 Sequence Detection System using $2 \mu \mathrm{L}$ transcriptase products as template under the conditions of $95^{\circ} \mathrm{C}$ for $15 \mathrm{~min}$, followed by 50 cycles of denaturation at $95^{\circ} \mathrm{C}$ for $30 \mathrm{~s}$, annealing, and extension at $60^{\circ} \mathrm{C}$ for $30 \mathrm{~s}$. The quantitative standard curve for determination of ORF095 copy number was created by real-time PCR of standard plasmid p095/EGFP serial 10-fold dilutions of a stock containing $10^{8}$ copies $/ \mu \mathrm{L}$. The specificity of the real-time PCR was confirmed by sequencing of the product. 


\section{Transfection and GTPV infection Vero cells}

Four siRNAs targeting ORF095 were designed to inhibit GTPV ORF095 gene expression in BHK-21 cells. We used a modified CMV promoter, a typical RNA polIII promoter, to drive the transcription of the siRNAs. To monitor the effects of the siRNAs, eukaryotic expression plasmid p095/EGFP was constructed, in which the ORF095 gene were fused to the 5'-end of the EGFP coding sequence, and cotransfected with their specific siRNA expression plasmids. So the inhibitory effects of the ORF095-specific siRNAs on the ORF095 expression could be indirectly evaluated by the expression of EGFP in the transfected cells.

To test whether the expressed siRNAs inhibited GTPV production, we first assessed the growing capacity of GTPV in Vero cells expressing siRNAs. Vero cells were seeded in six-well plates and cultured at $37^{\circ} \mathrm{C}$ and $5 \%$ $\mathrm{CO}_{2}$ overnight. When the cell reached $70-80 \%$ confluence, $2.5 \mu \mathrm{g}$ of p61/GFP, p70/GFP, p165/GFP or p296/ GFP each were cotransfected with an equal amount of $\mathrm{pC} / \mathrm{GFP}$ as described above, respectively. The nonspecific vector $\mathrm{pC} / \mathrm{GFP}$ and non-transfected Vero cells were also used as a control. $24 \mathrm{~h}$ posttransfection, the cells were infected with GTPV at a multiplicity of infection (moi) of about 0.01. Briefly, after removing the culture medium, GTPV $(200 \mu \mathrm{l})$ in infection medium $(2.5 \mu \mathrm{g} / \mathrm{ml}$ trypsin), respectively, were added to each well. The cultures were then incubated at $37^{\circ} \mathrm{C}, 5 \% \mathrm{CO}_{2}$ for $4 \mathrm{~h}$, at which point the culture medium was replaced with fresh DMEM containing $2 \%$ fetal bovine serum.

\section{Virus titration}

To determine transfection efficiency, we monitored GFP fluorescence intensity of transfected cells using fluorescent microscope analysis. Culture supernatants were collected for virus titration. Six days post infection, supernatants was harvested from the infected cultures and virus titer (TCID50) was determined three times on Vero cells.

Virus infectivity was determined by serial dilutions of the samples in 96-well plates and the virus titer was calculated as a TCID50 by the Reed-Muench method [28]. A viral suspension titrated at $10^{-1}$ to $10^{-8}$ TCID50 per $0.1 \mathrm{ml}$ was used for viral challenge. Vero cells (about $80 \%$ confluent) grown in 96 -well plates were transiently transfected with $0.1 \mu \mathrm{g}$ p61/GFP, p70/GFP, p165/GFP and p296/GFP, respectively, per well. After $6 \mathrm{~h}$ of transfected, the transfection medium was removed and the cells were washed twice with DMEM medium. The transfected cells were then infected with 100 TCID50 of virus per $0.1 \mathrm{ml}$ per well. After additional $1 \mathrm{~h}$ incubation, the inoculum was removed and the cells were washed twice with DMEM medium. The cells were then maintained in DMEM medium supplemented with $10 \%$ fetal bovine serum for 6 days. For detecting the therapeutic potential of siRNA, in another parallel experiment, transfection was performed $1 \mathrm{~h}$ post-infection with the virus. GTPV replication in Vero cells was evaluated by virus titer (TCID50).

\section{Results}

Transient cellular transfection and analysis of the targeted gene and EGFP expression in BHK-21 cells

Different siRNAs suppressed the expression of fusion green fluorescent protein in BHK-21 cells is different. The siRNAs targeting to the conserved region of GTPV genome were generated in vitro by human recombinant Dicer enzyme, as described in Figure 1B. To identify an effective inhibitory effect of siRNAs, the cDNA cassettes of these regions were inserted into the 5' end of enhanced green fluorescent protein (EGFP) gene to construct reporter plasmids (Figure 2). The reporter plasmids were used to cotransfect BHK-21 cells with either the homologous siRNAs or the heterologous siRNAs. The results showed that the number of EGFP-expressing cell was markedly reduced in the sample transfected with homologous siRNAs than sample transfected with heterologous siRNAs or non-transfected (Figure 3A). FACS demonstrated that the levels of inhibition mediated by the siRNAs were similar among the different experiment groups and significantly higher than the control group (cotransfection with heterologous siRNAs or without siRNAs).

The inhibitory effects of the siRNAs on expression of EGFP were quantitatively validated by flow cytometry 48 $h$ posttransfection. The extent of EGFP down regulation was assessed by the mean fluorescence of the positive cells (LR-values) and the rate of EGFP positive cells

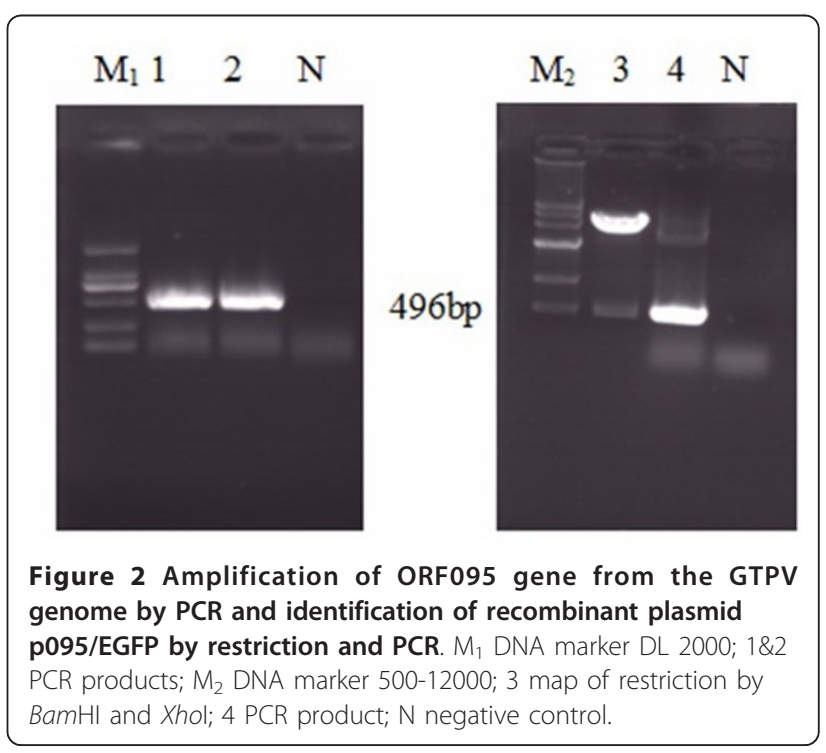


(A)
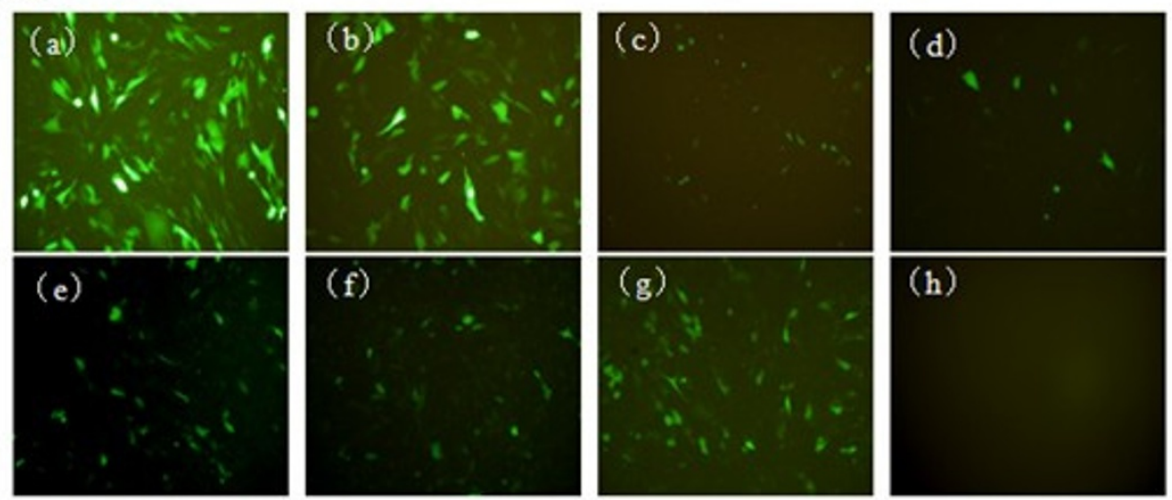

(B)
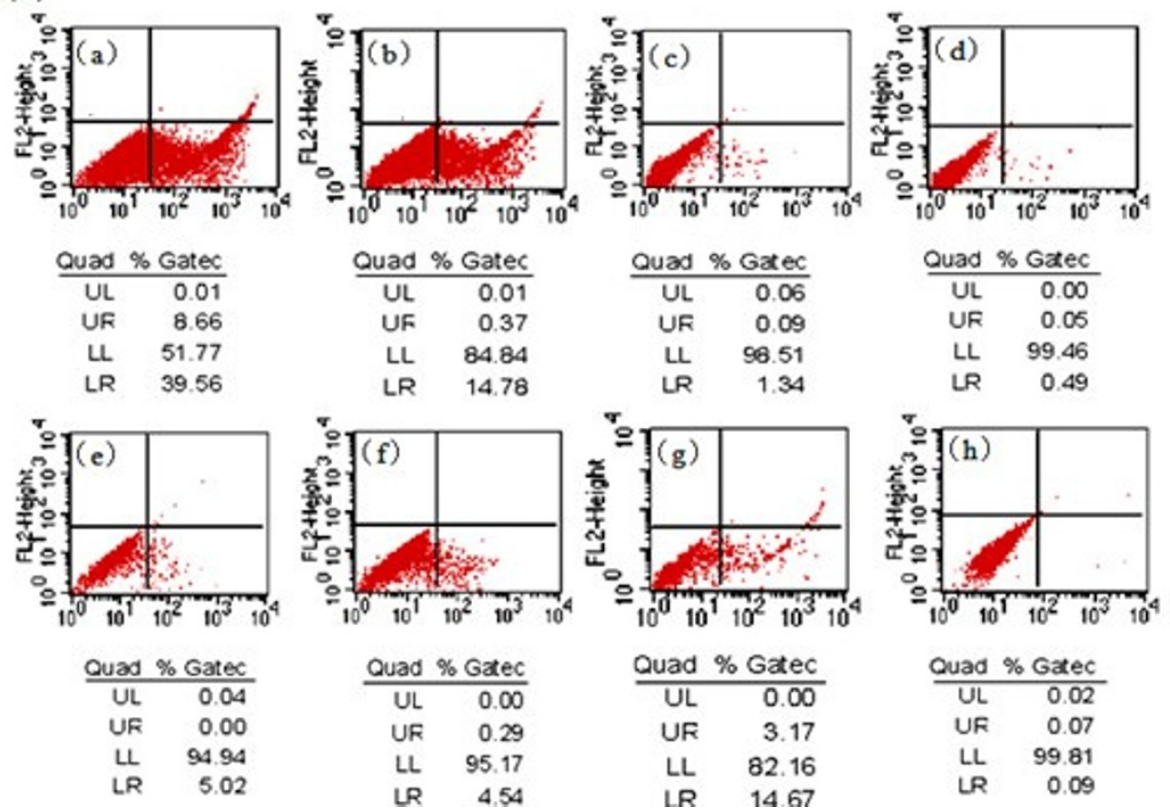

\begin{tabular}{cr} 
Quad & Gatec \\
\hline UL & 0.02 \\
UR & 0.07 \\
LL & 99.81 \\
LR & 0.09
\end{tabular}

Figure 3 Transient expression of siRNAs conferred the sequence-specific inhibition of expression of GTPV ORF95 in BKH-21 cells. (A) Fluorescence detection of cotransfection of p095/EGFP with their corresponding siRNA expression plasmids $24 \mathrm{~h}$ posttransfection. (B) Flow cytometric analysis of cotransfection of p095/EGFP with their corresponding siRNA expression plasmids $48 \mathrm{~h}$ posttransfection. EGFP expression level in cells cotransfected with (a) pEGFP-N1 vector; (b) p095/EGFP; (c) p095/EGFP and p61; (d) p095/EGFP and p70; (e) p095/EGFP and p165; (f) p095/EGFP and p296; (g) p095/EGFP and pControl; (h) BHK-21 cell control. LR-Value means the rate of EGFP positive cells.

(Figure 3B). Compared with the $\mathrm{pC}$, the LR-values of the EGFP positive cells were reduced in the cells transfected with ORF095 siRNA-specific expression plasmids p61, p70, p165 and p296, and were reduced by $90.9 \%, 96.7 \%$, $66.0 \%$ and $69.32 \%$, respectively.

To further demonstrate the levels of inhibition, cells were collected $48 \mathrm{~h}$ post-transfection and RT-PCR analysis was performed. The level of target RNA, as determined by RT-PCR, was also significantly reduced in cells transfected with homologous siRNAs (Figure 4A). To measure the level of gene suppression accurately, QPCR primers and Taqman probes directing to ORF095 were designed. We also designed probes and primers directed to $\beta$-actin sequence (serve as internal reference). When normalized for loading differences using the $\beta$ actin mRNA, the ORF095 message in the cells transfected with p61, p70, p165 and p296 were reduced by $69 \%$, 97\%, 22\%and 57\% (ORF095 message copies ratios of cells transfected with shRNA expression vectors/cells transfected with empty vector)(Figure $4 \mathrm{~B}$ ). There was no significant inhibition in cells transfected with the empty vector $\mathrm{pEGFP}-\mathrm{N} 1$ and nonspecific shRNA expression vector pC. mRNA levels of ORF095 (average ORF095 mRNA levels in cells treated with p61, p70, p165, p296, $\mathrm{pC}$ and empty vector were $0.416,0.036,1.046,0.580$, 1.345 , respectively) or $\beta$-actin suggested that the 


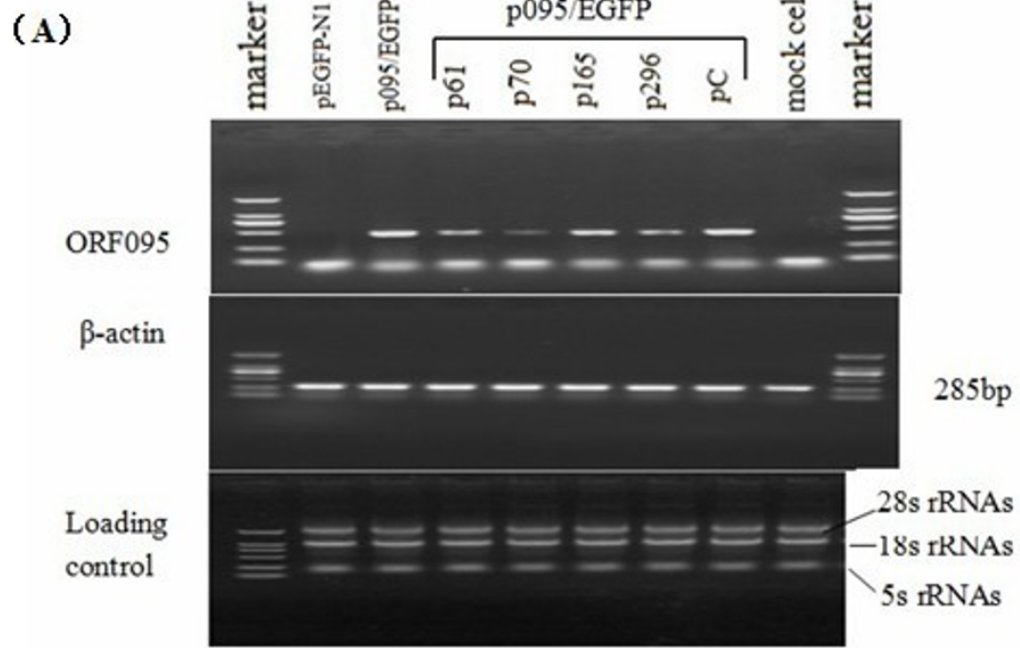

(B)

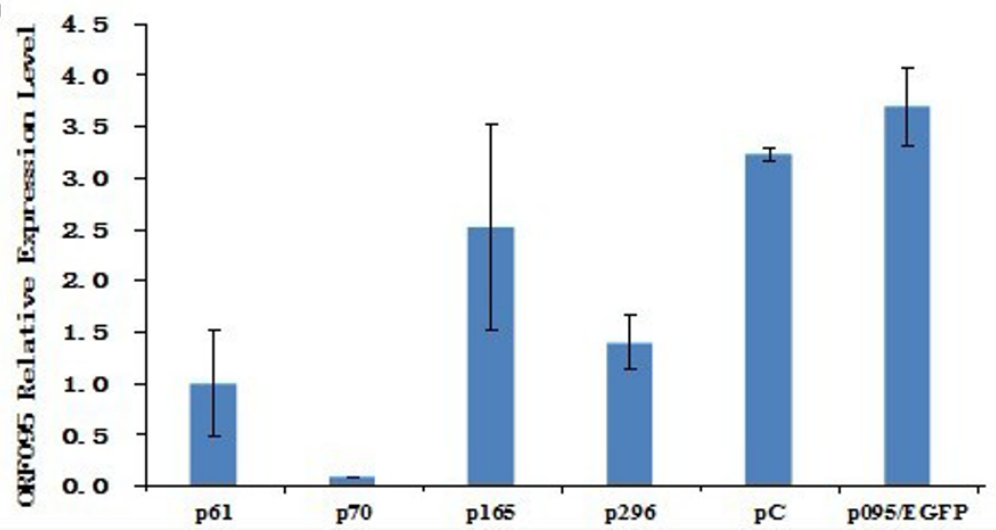

Figure 4 Analysis effect of RNAi on ORF095 by RT-PCR. (A) Cells were harvested, and transcripts were analyzed by RT-PCR amplification. The 28S, 18S, and 5.8S rRNAs were visualized under UV light for equal loading control. (B) Specific siRNA inhibits the accumulation of ORF095 message. BHK-21 cells were transfected with pEGFP-N1 and then transfected with variant shRNA-expressing vectors. Forty hours posttransfection, total RNA was extracted and subjected to fluorescence quantitative PCR analysis. pC transfected cells and mock-transfected cells were used as controls. The mRNA of beta actin served as an internal reference.

reductions in ORF095 message did not result from poor transfection, nonspecific inhibition or toxicity, because the average mRNA levels of $\beta$-actin for experimental cells were not significantly reduced compared to the control cells. In addition, the suppressive effect was found to be gene-specific, because the inhibitory effect of empty vector and nonspecific shRNA expression vector $\mathrm{pC}$ were negligible. These results suggest that the siRNA generated by in vitro transcription effectively and specifically inhibit the expression of GTPV ORF095 conserved regions in BHK-21 cells.

\section{Interference of GTPV replication by shRNA expression vector}

To investigate whether or not knockout of ORF095 relieves cytopathic effect (CPE) induced by GTPV, Vero cells were transfected by plasmids expressing
ORF095 protein-targeted shRNAs (p61/GFP, p70/GFP, p165/GFP and p296/GFP), respectively. Nonspecific shRNA expression vector $\mathrm{pC} / \mathrm{GFP}$ was transfected in parallel. $4 \mathrm{~h}$ post-transfection, the cultures were infected with GTPV and checked daily. Six days later, we found that cells pre-transfected with p70/GFP exhibited less CPE, whereas other shRNA-treated cells and the empty vector control demonstrated the same typical GTPV-induced CPE as cells infected only with virus, as shown in Figure 5.

The TCID50 assay was performed to examine the effect of siRNA on production of viable virus, and the results (Figure 6) showed that in control cells the titers reached $10^{-5.12}$ TCID50/0.1 mL at 6 days post-infection. In contrast, the titers in the cells transfected with p61/ GFP, p70/GFP, p165/GFP or p296/GFP, were $10^{-3.75}$, $10^{-2.48}, 10^{-4.73}$ and $10^{-4.66} / 0.1 \mathrm{~mL} 6$ days post-infection 


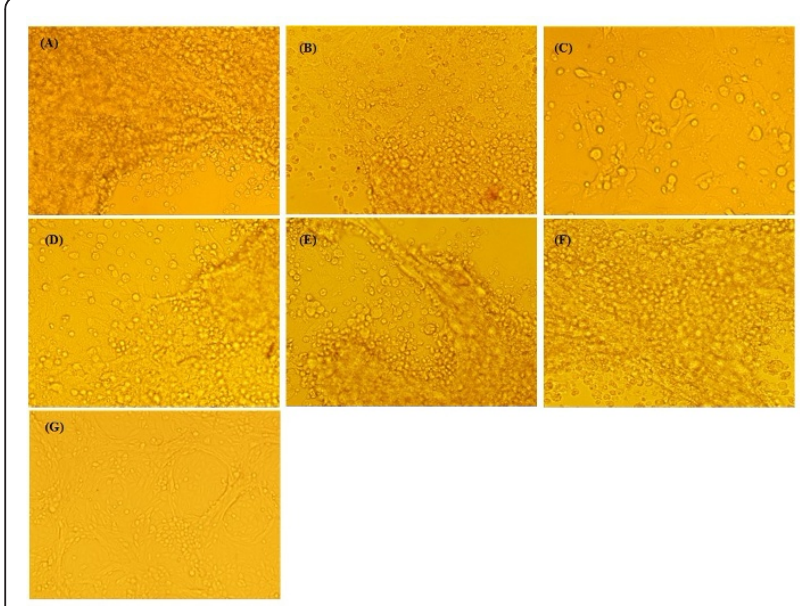

Figure 5 Cytopathic effect (CPE) analysis of GTPV on Vero cells transfected with shRNA-expressing vectors ( $2.5 \mu \mathrm{g}$ each). (A): Cells just were infected GTPV; (B)-(G):p61/GFP, p70/GFP, p165/GFP, p296/GFP, pC/GFP and untreated cells, respectively. Except(G), all cells were infected with GTPV(A/Goat/Qinghai/SV40/2006) isolate at a multiplicity of infection (MOI) of 0.01 . Pictures were taken at 6 days post-infection with an Olympus digital camera (Olympus, Japan) at a magnification of $\times 40$ with an exposure time of $1 / 8 \mathrm{~s}$.

respectively, corresponding to 23.4-, 463.5-, 1.4- and 2.9 - fold reductions in viable virus production.

\section{Dose-dependent inhibitory effect of shRNA expression vector p70/GFP}

To characterize the antiviral properties of most potent siRNA construct, namely p70/GFP, Vero cells were transfected with dilution of each construct to cover a range of 0.1-5.0 $\mathrm{g}$ in six concentrations. Overnight transfected cells were infected with GTPV at an MOI of 0.01 and viral replication was examined at $48 \mathrm{~h}$ PI

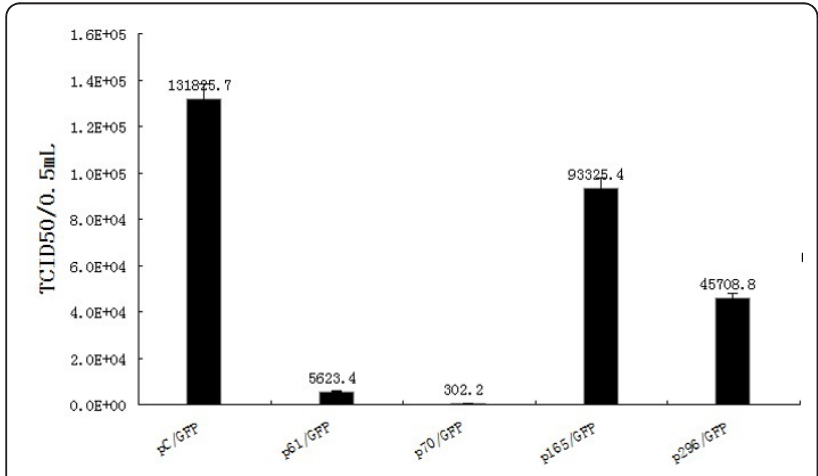

Figure 6 GTPV-ORF095 gene RNA-specific siRNAs can inhibit the production of progeny virus. Vero cells transiently transfected with empty vector or plasmid carry siRNA-61, 70, 165 or 296 were infected with GTPV at an MOI of 0.01. Viruses were harvested and titers were determined as described above. Mock-infected cells were used as a control. The values given are average of three independent experiments. Error bars indicate standard deviations.

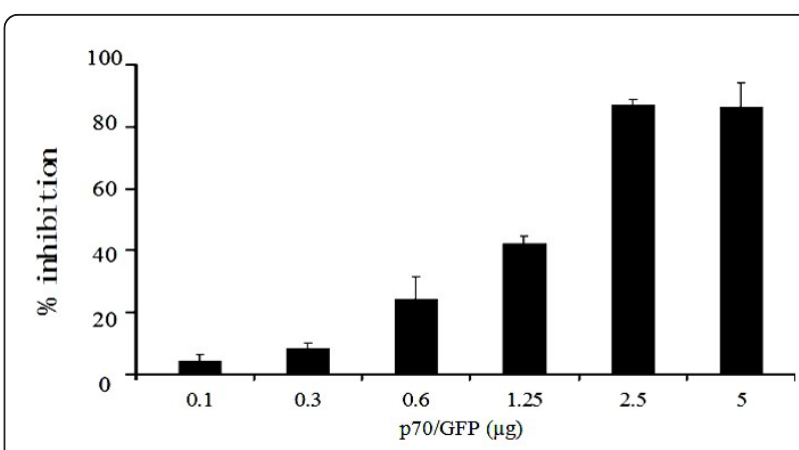

Figure 7 Dose-dependent inhibitory effect of shRNA expression vector p70/GFP. Vero cells were transfected with variant p70/GFP and then infected with GTPV at an $\mathrm{MOI}$ of 0.01. 48 h post-infection, total RNA was extracted and subjected to quantitative PCR analysis. Mock-transfected cells were used as controls. The mRNA of beta actin served as an internal reference. Black bars indicate normalized shRNA+ (cells transfected with p70/ GFP at six different concentrations ranging from 0.1 to $5.0 \mu \mathrm{g}$ ) GTPV message copies ratios. The data shown represent average from three experiments with the standard deviations indicated by error bars.

(Figure 7). p70/GFP showed average viral replication inhibition of $5.3 \%$ at the lowest tested concentration of $0.1 \mu \mathrm{g}$, and complete inhibition at concentrations of 2.5 $\mu \mathrm{g}$ and higher.

\section{Discussion}

RNAi is a process of sequence-specific, post-transcriptional gene silencing that is initiated by double stranded RNA. Introduction of siRNA results in degradation of siRNA specific transcripts thus reducing the expression of their protein product. In plants, it is a natural antiviral defense mechanism [29]. In mammalian cells, however, dsRNAs longer than $30 \mathrm{nt}$ activate an antiviral defense leading to the nonspecific degradation of RNA transcripts and a general shutdown of host-cell protein translation [30]. The successful use of siRNA in animal cells encouraged the development of siRNA expression vector [31] and numerous studies have demonstrated that DNA-based siRNA is a promising approach for antiviral therapy in mammals. RNAi represents a new antiviral method and is being increasingly used to inhibit the replication of viral pathogens [15], such as HIV1 [32], hepatitis C [33], influenza [34], severe acute respiratory syndrome [35] and hepatitis E viruses [36]. This study has demonstrated the use of pGPU6/Neo or pGPU6/GFP/Neo vector-based RNAi against GTPV, a major pathogen of goats and sheep. Four different siRNA targeting viral gene ORF095, one key gene involved in GTPV replication, successfully reduced viral replication.

The results showed that the ORF095-specific siRNAs p70 could effectively down-regulate the expression of 
ORF095, while p61, p165 and p296 displayed weak activity. Additionally, expression of the housekeeping gene $\beta$-actin was also analyzed by RT-PCR and quantitative real-time PCR, and no significant difference in the expression of $\beta$-actin was observed between the siRNAs treatment groups and $\mathrm{pC}$ treatment groups.

Different siRNA sequences display widely different efficacies with regard to suppression of gene expression, requiring screening of multiple sequences [37]. In this research, we have selected four target sequences for RNA interference by the software applications, "siRNA Target Finder and Design Tool" available at http://www.ambion. com/. As the ORF095 gene is well conserved in GTPV and ORF095 protein is a virion core protein and assembly protein in GTPV, we selected ORF095 as a target gene. In order to generate shRNA expression cassettes quickly and accurately, we employed a PCR-based strategy to clone siRNA sequences. In this strategy, siRNA sequences were designed as a single primer sequence of which $19 \mathrm{bp}$ complementary to the human U6 promoter were added. The resulting PCR products are shRNA expression cassettes including the human U6 promoter. shRNAs that have been generated from this expression system are efficiently processed by dicer into siRNAs. In addition, in this study, we selected pEGFP-N1 vector that contains an EGFP gene as report gene and can be transfected into mammalian cells using any standard transfection method.

Vero cells transfected with p61/GFP, p70/GFP, p165/ GFP, p296/GFP and pC/GFP were examined for CPE by virus titration. All results demonstrated that siRNA-70 is the most effective one, and result showed that minimum concentration of the construct p70/GFP is $2.5 \mu \mathrm{g}$ required to induce maximum inhibition.

Blasting ORF095 sequence in GenBank revealed that there were 8 Capripoxvirus isolates containing identical sequence corresponding to ORF095. In view of the sequences of the ORF095 genes of GTPV strains from the same genotype, they all share high homology (95$100 \%)$. Therefore, ORF095 gene is a good target to suppress GTPV replication by RNAi.

\section{Conclusion}

In conclusion, this study demonstrates that vector-based shRNA methodology can effectively inhibit GTPV replication on Vero cells. Further study will be required to determine whether such treatment protect against GTPV infection in vivo. Still, this work represents a strategy for controlling goatpox, potentially facilitating new experimental approaches in the analysis of both viral and cellular gene functions during of GTPV infection.

\section{Abbreviations}

GTPV: Goatpox virus; RNAi: RNA-mediated interference; siRNA: Short interference RNA; shRNA: Short hairpin RNA; GFP: Green fluorescent protein;
EGFP: Enhanced green fluorescent protein; p095/EGFP: pEGFP-N1 expression ORF095 gene cassette; p61 p70, p165, p296 and pC were siRNA-61, 70, 165, 196 and control expression cassettes pGU6/Neo plasmids; p61/GFP p70/GFP: p165/GFP, p296/GFP and pC/GFP were siRNA-61, 70, 165, 196 and control expression cassettes pGU6/GFP/Neo plasmids; CPE: Cytopathic effect; PCR: Polymerase chain reaction; RT-PCR: Retro transcription PCR; SPPV: Sheeppox virus; LSDV: Lumpy Skin Disease Virus; MYXV: Myxoma virus; VACV: Vaccinia virus; BHK-21: Baby Hamster Kidney cells; Vero: African green monkey kidney cells; DMEM: Dulbecco's modified Eagle's medium; FBS: Fetal bovine serum; FACS: Fluorescence-activated cell sorting; PBS: Phosphate-buffered saline; MOI: Multiplicity of infection; PI: Post-infection.

\section{Acknowledgements}

We thank Yadong Zheng for thoughtful discussions and assistance during manuscript preparation. The study was supported by grants from the China's Ministry of Agriculture Genetically Modified Organisms Breeding Technology Major Program (no. 2009ZX08008-010B) and the National Natural Science Foundation of China (no.31001056-C1803).

\section{Author details}

${ }^{1}$ Key Laboratory of Animal virology of the Ministry of Agriculture, State Key Laboratory of Veterinary Etiological Biology, Lanzhou Veterinary Research Institute, Chinese Academy of Agriculture Sciences, No. 1 Xujiaping, Lanzhou, Gansu 730046, PR China. 'Lanzhou Veterinary Research Institute, Chinese Academy of Agriculture Sciences, No. 1 Xujiaping, Lanzhou, Gansu 730046, PR China.

\section{Authors' contributions}

QZ and XC designed research; ZZ performed research and wrote the paper; GW helped to construct partial plasmids and analyzed data; XZ contributed new reagents/analytic tools; $X Y$ provided partial plasmids. $\mathrm{HZ}$ helped to culture cells; JL helped to culture viruses; YX D had a co-ordination role in this work. All authors read and approved the final manuscript.

\section{Competing interests}

The authors declare that they have no competing interests.

Received: 15 August 2011 Accepted: 17 February 2012 Published: 17 February 2012

\section{References}

1. Nawathe DR, Asagba MO, Abegunde A, Ajayi SA, Durkwa L: Some observations on the occurrence of lumpy skin disease in Nigeria. Zentralb/ Veterinarmed B 1982, 29:31-36.

2. Carn VM: Control of capripoxvirus infections. Vaccine 1993, 11:1275-1279.

3. Tulman ER, Afonso CL, Lu Z, Zsak L, Sur JH, Sandybaev NT, Kerembekova UZ, Zaitsev VL, Kutish GF, Rock DL: The genomes of sheeppox and goatpox viruses. J Virol 2002, 76:6054-6061.

4. Johnston JB, Barrett JW, Nazarian SH, Goodwin M, Ricciuto D, Wang G, McFadden G: A poxvirus-encoded pyrin domain protein interacts with ASC-1 to inhibit host inflammatory and apoptotic responses to infection. Immunity 2005, 23:587-598

5. Van Vliet K, Mohamed MR, Zhang L, Villa NY, Werden SJ, Liu J, McFadden G: Poxvirus proteomics and virus-host protein interactions. Microbiol Mol Biol Rev 2009, 73:730-749.

6. Kovacs GR, Moss B: The vaccinia virus $H 5 R$ gene encodes late gene transcription factor 4: purification, cloning, and overexpression. J Virol 1996, 70:6796-6802.

7. Maa JS, Esteban M: Structural and functional studies of a 39,000-Mr immunodominant protein of vaccinia virus. J Virol 1987, 61:3910-3919.

8. Sharp PA, Zamore PD: Molecular biology. RNA interference. Science 2000, 287:2431-2433.

9. Elbashir SM, Harborth J, Lendeckel W, Yalcin A, Weber K, Tuschl T: Duplexes of 21-nucleotide RNAs mediate RNA interference in cultured mammalian cells. Nature 2001, 411:494-498.

10. Sohail M, Doran G, Riedemann J, Macaulay V, Southern EM: A simple and cost-effective method for producing small interfering RNAs with high efficacy. Nucleic Acids Res 2003, 31:e38.

11. Brummelkamp TR, Bernards R, Agami R: A system for stable expression of short interfering RNAs in mammalian cells. Science 2002, 296:550-553. 
12. Pinkenburg O, Platz J, Beisswenger C, Vogelmeier C, Bals R: Inhibition of NF-kappaB mediated inflammation by siRNA expressed by recombinant adeno-associated virus. J Virol Methods 2004, 120:119-122.

13. Rubinson DA, Dillon CP, Kwiatkowski AV, Sievers C, Yang L, Kopinja J, Rooney DL, Zhang M, Ihrig MM, McManus MT, Gertler FB, Scott ML, Van Parijs L: A lentivirus-based system to functionally silence genes in primary mammalian cells, stem cells and transgenic mice by RNA interference. Nat Genet 2003, 33:401-406.

14. Zhao $L$, Jian $H$, Zhu H: Specific gene inhibition by adenovirus-mediated expression of small interfering RNA. Gene 2003, 316:137-141.

15. He YX, Hua RH, Zhou YJ, Qiu HJ, Tong GZ: Interference of porcine reproductive and respiratory syndrome virus replication on MARC-145 cells using DNA-based short interfering RNAs. Antiviral Res 2007, 74:83-91.

16. Haasnoot J, Westerhout EM, Berkhout B: RNA interference against viruses: strike and counterstrike. Nat Biotechnol 2007, 25:1435-1443.

17. Luo J, Du J, Gao S, Zhang G, Sun J, Cong G, Shao J, Lin T, Chang H: Lentviral-mediated RNAi to inhibit target gene expression of the porcine integrin av subunit, the FMDV receptor, and against FMDV infection in PK-15 cells. Virol J 2011, 8:428.

18. Chen W, Liu M, Jiao Y, Yan W, Wei X, Chen J, Fei L, Liu Y, Zuo X, Yang F, Lu Y, Zheng Z: Adenovirus-mediated RNA interference against foot-andmouth disease virus infection both in vitro and in vivo. J Virol 2006, 80:3559-3566

19. Yin R, Ding Z, Liu X, Mu L, Cong Y, Stoeger T: Inhibition of Newcastle disease virus replication by RNA interference targeting the matrix protein gene in chicken embryo fibroblasts. J Virol Methods 2010, 167:107-111.

20. Li J, Guo H, Shi Z, Tu C: In vitro inhibition of CSFV replication by retroviral vector-mediated RNA interference. J Virol Methods 2010 169:316-321.

21. Alkhalil A, Strand S, Mucker E, Huggins JW, Jahrling PB, Ibrahim SM: Inhibition of Monkeypox virus replication by RNA interference. Virol J 2009, 6:188

22. Brummelkamp TR, Bernards R, Agami R: Stable suppression of tumorigenicity by virus-mediated RNA interference. Cancer Cell 2002, 2:243-247

23. McCaffrey AP, Nakai H, Pandey K, Huang Z, Salazar FH, Xu H, Wieland SF, Marion PL, Kay MA: Inhibition of hepatitis $B$ virus in mice by RNA interference. Nat Biotechnol 2003, 21:639-644.

24. Miyagishi M, Taira K: U6 promoter-driven siRNAs with four uridine $3^{\prime}$ overhangs efficiently suppress targeted gene expression in mammalian cells. Nat Biotechnol 2002, 20:497-500.

25. Paddison PJ, Caudy AA, Sachidanandam R, Hannon GJ: Short hairpin activated gene silencing in mammalian cells. Methods Mol Biol 2004 265:85-100.

26. Paul CP, Good PD, Winer I, Engelke DR: Effective expression of small interfering RNA in human cells. Nat Biotechnol 2002, 20:505-508.

27. Elbashir SM, Harborth J, Weber K, Tuschl T: Analysis of gene function in somatic mammalian cells using small interfering RNAs. Methods 2002, 26:199-213.

28. Reed $L$, Muench $H A$ : A simple method of estimating fifty percent end points. Am J Hyg 1938, 27:493-497.

29. Goff A, Twenhafel N, Garrison A, Mucker E, Lawler J, Paragas J: In vivo imaging of cidofovir treatment of cowpox virus infection. Virus Res 2007. 128:88-98.

30. Nilsen TW, Maroney PA, Baglioni C: Maintenance of protein synthesis in spite of mRNA breakdown in interferon-treated HeLa cells infected with reovirus. Mol Cell Biol 1983, 3:64-69.

31. Miyagishi $M$, Taira K: Development and application of siRNA expression vector. Nucleic Acids Res Supp/ 2002, 2:113-114

32. Novina CD, Murray MF, Dykxhoorn DM, Beresford PJ, Riess J, Lee SK Collman RG, Lieberman J, Shankar P, Sharp PA: siRNA-directed inhibition of HIV-1 infection. Nat Med 2002, 8:681-686.

33. Sen A, Steele R, Ghosh AK, Basu A, Ray R, Ray RB: Inhibition of hepatitis C virus protein expression by RNA interference. Virus Res 2003, 96:27-35.

34. Ge Q, Filip L, Bai A, Nguyen T, Eisen HN, Chen J: Inhibition of influenza virus production in virus-infected mice by RNA interference. Proc Natl Acad Sci USA 2004, 101:8676-8681.

35. Akerstrom S, Mirazimi A, Tan YJ: Inhibition of SARS-CoV replication cycle by small interference RNAs silencing specific SARS proteins, 7a/7b, 3a/ $3 \mathrm{~b}$ and S. Antiviral Res 2007, 73:219-227.
36. Huang F, Hua X, Yang S, Yuan C, Zhang W: Effective inhibition of hepatitis E virus replication in A549 cells and piglets by RNA interference (RNAi) targeting RNA-dependent RNA polymerase. Antiviral Res 2009, 83:274-281.

37. Amarzguioui M, Rossi JJ, Kim D: Approaches for chemically synthesized siRNA and vector-mediated RNAi. FEBS Lett 2005, 579:5974-5981.

doi:10.1186/1743-422X-9-48

Cite this article as: Zhao et al.: RNA interference targeting virion core protein ORF095 inhibits Goatpox virus replication in Vero cells. Virology Journal 2012 9:48.

\section{Submit your next manuscript to BioMed Central and take full advantage of:}

- Convenient online submission

- Thorough peer review

- No space constraints or color figure charges

- Immediate publication on acceptance

- Inclusion in PubMed, CAS, Scopus and Google Scholar

- Research which is freely available for redistribution
C Biomed Central 